\title{
Prediction of Left Ventricular Ejection Fraction Using Simple
}

\section{Quantitative Clinical Information}

KEMP B. CEASE, M.D.*

JOHN M. NICKLAS, M.D.

Ann Arbor, Michigan

From the Cardiology Division, Department of Internal Medicine, the University of Michigan, Ann Arbor, Michigan. Requests for reprints should be addressed to Dr. John M. Nicklas, Cardiology Division, 3910 Taubman, University of Michigan Hospital, Ann Arbor, Michigan 48109. Manuscript submitted June 8, 1985, and accepted September 6, 1985.

${ }^{*}$ Current address: Building 10, Room 6B12, National Institutes of Health, Bethesda, Maryland 20892.
The left ventricular ejection fraction is useful in characterizing cardiac performance and evaluating prognosis in patients with known or suspected cardiac disease. The purpose of this study was to determine if simple, quantitative clinical information generated as part of a routine patient evaluation could be used to predict ejection fraction determined by radionuclide ventriculography. Multiple regression analysis was used to study a group of 64 patients selected to represent the full range of ejection fraction values. All patients had undergone cardiac catheterization and standard chest radiography in addition to resting and exercise radionuclide ventriculography. Using easily determined clinical variables, a regression formula was developed that predicted the radionuclide ventriculographic ejection fraction $(r=0.73)$. Plain film heart volume, heart rate, pulse pressure, and thoraclc width were highly significant terms in the optimal regression equation. For validation, the formula was applied to a second, independent verification data set composed of 41 cases and revealed similar correlation $(r=0.78)$. A radionuclide ventriculographic ejection fraction below 40 was identified in the verification data set with a sensitivity of 87 percent and specificity of 83 percent. Use of this method, requiring only direct heart rate, blood pressure, and chest radiographic measurements and simple calculations, may assist physicians in patient management and facilitate the optimal use of more invasive and expensive studles.

The left ventricular ejection fraction is well established as an important clinical index of the contractile state of the heart. It is of substantial prognostic value in patients with acute myocardial infarction [1-4], chronic coronary artery disease $[5,6]$, valvular heart disease $[5,7,8]$, and cardiomyopathy [9]. However, determination of ejection fraction by angiographic or radionuclide ventriculography or estimation using other techniques is attended by varying degrees of inconvenience, invasiveness, or expense. Information from the history, physical examination, electrocardiography, and chest radiography has been shown to correlate with ejection fraction in selected patients [10-13]. However, no generally useful method for estimating ejection fraction from routine clinical data has been demonstrated. In this report, we present a generally applicable method for predicting left ventricular ejection fraction derived from a physiologically based model using routine, quantitative clinical data.

\section{PATIENTS AND METHODS}

Model Development. The fundamental equations relating cardiac output (CO), stroke volume (SV), heart rate (HR), left ventricular ejection fraction (EF), left ventricular end-diastolic volume (EDV), perfusion pressure (P), and systemic vascular resistance (SVR) served as the foundation for the model: 
TABLE I Stratification of Exploratory Data Set

\begin{tabular}{|c|c|c|c|}
\hline & Number & $\begin{array}{c}\text { Ejection } \\
\text { Fraction } \\
(\text { mean } \pm S D)\end{array}$ & r Value* \\
\hline \multicolumn{4}{|l|}{ Sex } \\
\hline Male & 41 & $44 \pm 24$ & 0.62 \\
\hline Female & 23 & $56 \pm 25$ & 0.81 \\
\hline \multicolumn{4}{|c|}{ Coronary artery disease $^{\dagger}$} \\
\hline None & 15 & $45 \pm 29$ & $0: 84$ \\
\hline Low-grade & 14 & $53 \pm 20$ & 0.58 \\
\hline \multicolumn{4}{|c|}{ Significantly diseased vessels } \\
\hline One & 14 & $49 \pm 21$ & 0.61 \\
\hline Two & 12 & $45 \pm 25$ & 0.59 \\
\hline Three & 3 & $54 \pm 26$ & 0.68 \\
\hline Four & 1 & 75 & - \\
\hline \multicolumn{4}{|c|}{ Maximal exercise level } \\
\hline Rest & 7 & $50 \pm 19$ & 0.72 \\
\hline 25 watts & 11 & $54 \pm 30$ & 0.90 \\
\hline 50 watts & 14 & $50 \pm 25$ & 0.58 \\
\hline 75 watts & 20 & $45 \pm 25$ & 0.85 \\
\hline 100 watts & 11 & $43 \pm 25$ & 0.47 \\
\hline 125 watts & 1 & 18 & - \\
\hline \multicolumn{4}{|c|}{ Valvular disease } \\
\hline \multicolumn{4}{|c|}{ Mitral regurgitation } \\
\hline None & 51 & $50 \pm 24$ & 0.69 \\
\hline Mild & 10 & $34 \pm 27$ & 0.92 \\
\hline Moderate & 3 & $45 \pm 30$ & 0.30 \\
\hline Severe & 0 & - & - \\
\hline \multicolumn{4}{|c|}{ Aortic regurgitation } \\
\hline None & 58 & $48 \pm 45$ & 0.73 \\
\hline Mild & 1 & 89 & - \\
\hline Moderate & 3 & $53 \pm 27$ & - \\
\hline Severe & 2 & $48 \pm 42$ & - \\
\hline \multicolumn{4}{|c|}{ Aortic stenosis } \\
\hline None & 60 & $45 \pm 13$ & 0.72 \\
\hline Mild & 1 & 89 & - \\
\hline Moderate & 1 & 80 & - \\
\hline Severe & 2 & $80 \pm 1$ & - \\
\hline \multicolumn{4}{|c|}{ Global hypokinesis } \\
\hline None & 34 & $68 \pm 13$ & 0.33 \\
\hline Mild & 10 & $38 \pm 16$ & 0.70 \\
\hline Moderate & 7 & $33 \pm 8$ & 0.95 \\
\hline Severe & 13 & $17 \pm 6$ & 0.56 \\
\hline
\end{tabular}

* Correlation between predicted and actual ejection fraction.

+ Significant coronary disease was defined as 75 percent stenosis or more of a major coronary vessel or 50 percent stenosis or more of the left main coronary artery.

$\mathrm{CO}=\mathrm{SV} \times \mathrm{HR}=\mathrm{EF} \times \mathrm{EDV} \times \mathrm{HR}$ and $\mathrm{CO}=\mathrm{P} / \mathrm{SVR}$. The right sides of these relationships were equated, and the resultant equation was solved for ejection fraction, yielding: $E F=P /(E D V \times H R \times S V R)$. Since the variables on the right side of the equation, with the exception of the heart rate, cannot be precisely determined during the routine clinical evaluation, the ejection fraction cannot be calculated directly. However, by generalizing the terms using easily determined clinical correlates for each and weighting the terms by assignment of undetermined exponents, the model assumes the form: $E F=$ (constant) $\times$ (pressure $)^{p} \times$

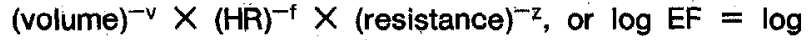
(constant) $+p \log$ (pressure) $-v \log ($ volume $)-f \log (\mathrm{HR})$ $-z \log$ (resistance). In this form, the optimal correlates for each term can be selected and the coefficients can be determined using standard multiple linear regression analysis.

Possible clinical correlates considered for each term included systolic and diastolic blood pressures and calculated mean arterial pressure and pulse pressure for the pressure term and cardiac measurements from posteroanterior and lateral chest radiography for the volume term [13]. Cardiac length is measured from the junction of the superior vena cava and right atrium to the cardiac apex. The broad diameter, is measured from the junction of the right atrium and the hemidiaphragm to the junction of the pulmonary artery and left atrial appendage. The depth is measured as the greatest horizontal depth of the heart on the lateral film. The width is the widest transverse width of the heart on the posteroanterior film. The thoracic width represents the widest transverse measurement of the thorax above the insertions of the diaphragms on the posteroanterior film. The calculated indexes of heart volume, plain film heart volume, and cardiothoracic ratio were also considered. The plain film heart volume is determined by multiplying 0.42 by the cardiac length by the cardiac broad diameter by the cardiac depth, as previously described [13]. Age was considered a possible correlate with systemic vascular resistance. Body size was considered as a possible correlate with either left ventricular end-diastolic volume or systemic vascular resistance, and thus the indicators height, weight, calculated body surface area, and thoracic width were considered [14].

Patients. In order to develop a model for predicting ejection fraction with validity over the full range of ejection fractions, a data set was required composed of patients with ejection fraction distributed relatively evenly throughout the range of interest. To allow subgroup analysis, structurally and functionally well-characterized patients were required. Therefore, all adult patients who had undergone cardiac catheterization from May 1979 through December 1982 and had resting and exercise radionuclide ventriculography during the same hospitalization and standard posteroanterior and lateral chest radiography within two weeks of ventriculography were considered for inclusion in the study. This population had a skewed normal distribution of ejection fractions, with a mode between 60 and 70 percent. To create a data set more evenly distributed by ejection fraction, the ejection fraction range from 0 to 100 was divided into 10 strata of equal width. All patients from 1981 were included as a core, and patients from other years were randomly recruited into the less populated strata until an even distribution was achieved or the patient pool was exhausted. In order to fill out the less populated strata at the extremes of the ejection fractlon distributlon, all resting and exercise ventriculographic results from January 1979 to December 1982 were reviewed, and additional patients with ejection fractions in the strata of interest, chest radiography within two weeks of radionuclide ventriculography, and cardiac catheterization at any time at the Unlversity of Michigan were included.

During the period, 2,727 patients underwent cardiac catheterization, of which 216 underwent resting and exer- 
cise radionuclide ventriculography during the same hospitalization. Recruitment of 124 patients from this group along with 29 patients in the extreme ejection fraction strata with non-concomitant catheterization yielded 153 patients in the initial data set, with 68 who were found to have standard posteroanterior and lateral chest radiography within two weeks of radionuclide ventriculography. No patients were excluded on the basis of technical or anatomic features of posteroanterior and lateral chest radiography. Four patients lacked documentation of the resting diastolic blood pressure at the time of ventriculography and were excluded. This final data set of 64 patients was termed the exploratory data set.

A second data set was created in a manner analogous to the first from patients undergoing resting and exercise radionuclide ventriculography from January through September 1983 who had posteroanterior and lateral chest radiography within two weeks of ventriculography. Cardiac catheterization was not required in this data set. Patients included in the exploratory data set were ineliglble for inclusion in this second data set. Fifty patients with concomitant catheterization were included as a core of the data set, and stratified recruitment was again used to recruit 74 additional patients. Of these 124 patients, 83 had radiology folders available and 41 had standard posteroanterior and lateral chest radiography within two weeks of radionuclide ventriculography. This second, independent, 41-patient data set was termed the verification data set.

Patient Evaluation. Radionuclide ventriculography at rest and with graded exercise was performed with patients in the supine position, and the ejection fraction was calculated from the background-corrected equilibrium time-activity curve using standard techniques. The resting ejection fraction, supine heart rate, and arm cuff blood pressure determined at the time of radionuclide ventriculography were used in this study. Cardiac catheterization was performed using standard procedures. Catheterization hemodynamic data were documented in all patients. All chest radiograms were standard upright six foot posteroanterior and lateral films. Significant coronary disease was defined as 75 percent or greater stenosis of a major coronary vessel or 50 percent or greater stenosis of the left main coronary artery. Statistical Analysis. Statistical analysis was performed employing the Michigan Interactive Data Analysis System (MIDAS) as currently implemented in the University of Michigan Terminal System using an Amdahl 5860 computer $[15,16]$. In the exploratory data set, stepwise multiple linear regression using variables and their logarithmic transformations was employed to examine the model as just described. To further evaluate the relative importance of using the physiologically based model, the simpler but nonphysiologic linear regression model using untransformed data was also examined and compared. Using the resultant formula from the physiologically based regression model, the correlation between the predicted and actual ejection fraction was examined in substrata in the exploratory data set. For independent validation, the formula was applied to the verification data set, and the correlation between the predicted and actual ejection fraction was examined. One predicted ejection fraction in the verification data set great-
TABLE II Univariant Correlations between Ejection Fraction or Log Ejection Fraction and Variables or Log Variables, Exploratory Data Set

\begin{tabular}{lrr}
\hline \multicolumn{1}{c}{ Variable } & $\begin{array}{c}\text { Correlation of } \\
\text { Variable with } \\
\text { Ejeclion Fraction* }\end{array}$ & $\begin{array}{c}\text { Correlation of } \\
\text { Log Variable with } \\
\text { Log Ejection Fraction* }\end{array}$ \\
\hline Cardiac length & -0.56 & -0.63 \\
Cardiac broad diameter & 0.55 & -0.61 \\
Cardiac depth & -0.37 & -0.39 \\
Cardiac width & -0.49 & -0.55 \\
Thoracic width & -0.17 & -0.14 \\
Cardiothoracic ratio & -0.42 & -0.50 \\
Plain film heart volume & -0.59 & -0.63 \\
Systolic blood pressure & 0.26 & 0.30 \\
Diastolic blood pressure & -0.03 & -0.09 \\
Pulse pressure & 0.35 & 0.49 \\
Mean arterial pressure & 0.20 & 0.06 \\
Heart rate & -0.60 & -0.61 \\
Height & -0.26 & -0.28 \\
Weight & 0.04 & 0.05 \\
Body surface area & 0.03 & 0.05 \\
Age & 0.33 & 0.44 \\
\hline * value. & &
\end{tabular}

* r value.

er than 100 was rounded to 100 . Test parameters were then calculated from the verification data set using standard definitions, with a positive result defined as a predicted ejection fraction below 40 [17]. A nomogram for graphic calculation of predicted ejection fraction was then developed on the basis of the derived formula and standard alignment chart techniques [18].

\section{RESULTS}

Characteristics of The Study Population. Stated indications for catheterization included chest pain (25 patients), coronary artery disease (seven), congestive heart failure (six), cardiomyopathy (five), recent myocardial infarction (four), syncope, arrhythmia, recent percutaneous transluminal angioplasty, and recent intracoronary streptokinase therapy (two each), endocarditis, possible mitral stenosis, mitral regurgitation, aortic stenosis, aortic insufficiency, pulmonary stenosis, pulmonary hypertension, myocardlal biopsy, and heart transplantation evaluation (one each). Other characteristics of the study population in the exploratory data set are shown in Table I.

Regression Analysis. The univariate correlation between ejection fraction or log ejection fraction and each of the variables studied is shown in Table II. Results of the multivariate analysis are shown in Table III. Stepwise multiple linear regression with the logarithmic transformed variables yielded an equation for log ejection fraction as a function of log plain film heart volume, log heart rate, log pulse pressure, and log thoracic width, with a multiple correlation coefficient of 0.83 , indicating that more than two thirds of the variability in log ejection fraction was explained by the equation $\left(R^{2}=0.68\right)$. The 
TABLE III Results of Stepwise Multiple Linear Regression Using Log-Transformed Varlables, Exploratory Data Set*

\begin{tabular}{lcccc}
\hline \multicolumn{1}{c}{ Variable } & Partial r Value & Coefficient & Standard Error & p Value \\
\hline Constant & & 11.513 & 2.407 & 0.0001 \\
$\begin{array}{c}\text { Log plain film } \\
\text { heart volume }\end{array}$ & -0.62126 & -1.2052 & 0.19790 & 0.0001 \\
$\begin{array}{c}\text { Log heart rate } \\
\text { Log pulse }\end{array}$ & -0.54875 & -1.4491 & 0.28741 & 0.0001 \\
$\quad \begin{array}{c}\text { pressure } \\
\text { Log thoracic }\end{array}$ & 0.35582 & 0.45141 & 0.15436 & 0.0049 \\
width & 0.32201 & 1.4621 & 0.55963 & 0.0114 \\
\hline
\end{tabular}

* Resultant equation after exponentiation: PEF $=10^{5} \times$ $\mathrm{PFHV}^{-1.2052} \times H R^{-1.4491} \times \mathrm{PP}^{0.45141} \times \mathrm{T}^{1.4621} ; \mathrm{r}=0.83, \mathrm{R}^{2}=$ 0.68 .

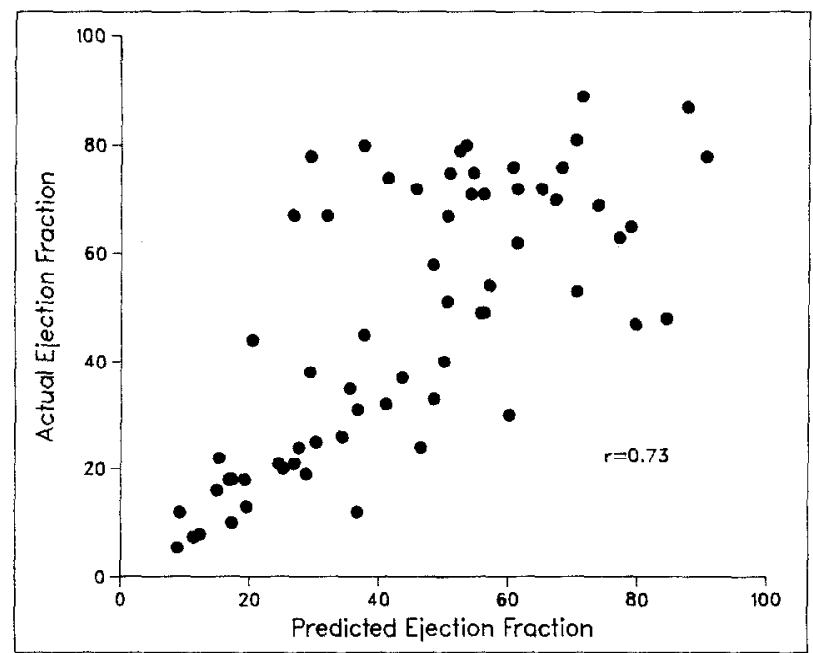

Flgure 1. Predicted ejection fraction by optimal nonlinear regression formula as compared with actual ejection fraction determined by radionuclide ventriculography in the exploratory data set.

logarithms of plain film heart volume (PFHV), heart rate $(\mathrm{HR})$, pulse pressure (PP), and thoracic width (T) were highly significant multivariate predictors of log ejection fraction, whereas all other variables were not. The coefficients, their standard deviations, and tests of significance of each term are as shown. To obtain an equation for use with nontransformed variables, both sides were exponentiated to yield the final equation for predicted ejection fraction (PEF): PEF $=10^{5} \times \mathrm{PFHV}^{-1.2052} \times \mathrm{HR}^{-1.4491} \times$ $\mathrm{PP}^{0.45141} \times \mathrm{T}^{1.4621}$. After the reverse transformation, good correlation between predicted and actual ejection fraction was preserved $(r=0.73)$. A plot of predicted and actual ejection fraction is shown in Figure 1.

Comparison of Models. The nature of the terms in the final regression model was predicted by the physiologic model, as was the sign of the exponent for plain film heart volume, heart rate, and pulse pressure. Examination of the relationship between predicted and actual ejection

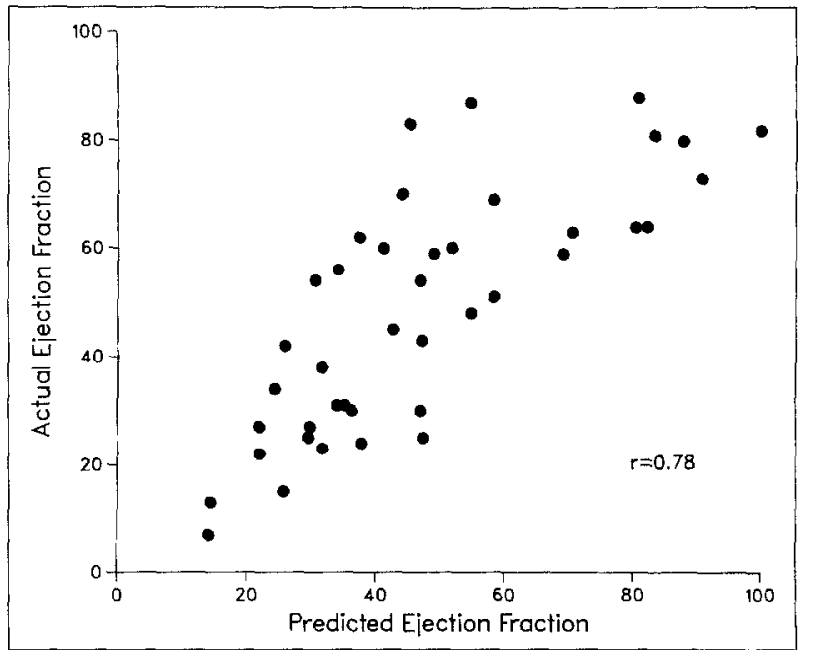

Flgure 2. Predicted and actual ejection fraction in the verification data set. For each patient in the verification data set, the ejection fraction was predicted using the formula derived in the exploratory data set and plotted against the actual value determined by radionuclide ventriculography. One patient had an ejection fraction of more than 100 , which was rounded to 100 , the theoretic limit of ejection fraction.

TABLE IV Summary of Test Parameters, Verification Data Set

\begin{tabular}{lcccc}
\hline Sensitivity & $87 \%$ & & \\
Specificity & $83 \%$ & & & \\
$\begin{array}{l}\text { Predictive values by } \\
\quad \text { pre-test likelihood of }\end{array}$ & & & & \\
$\begin{array}{l}\text { a positlve result } \\
\text { (ejection fraction below 40) }\end{array}$ & $10 \%$ & $50 \%$ & $90 \%$ & Ratios \\
Positive & 0.36 & 0.76 & 0.98 & 5.1 \\
Negative & 0.98 & 0.86 & 0.58 & 0.16 \\
\hline
\end{tabular}

fraction revealed increasing variability with increasing ejection fraction, as has been demonstrated previously with ejection fraction determined by ventriculography [19-22]. When stepwise multiple regression was performed using the simpler but nonphysiologic linear model, the variables plain film heart volume and heart rate were again selected ( $p<0.0001$ for each), whereas all other variables including pulse pressure and thoracic width were excluded ( $p<0.05)$. The correlation was similar to that of the physiologically based model $(r=0.72)$; however, the relationship between the actual ejection fraction and that predicted by the nonphysiologic model was distinctly nonlinear, as most clearly revealed by examination of the residuals. This suggested that the simpler underlying mathematical model was less appropriate and thus favored the additional complexity of the nonlinear physiologically based model.

Correlation within Substrata. The correlation within 


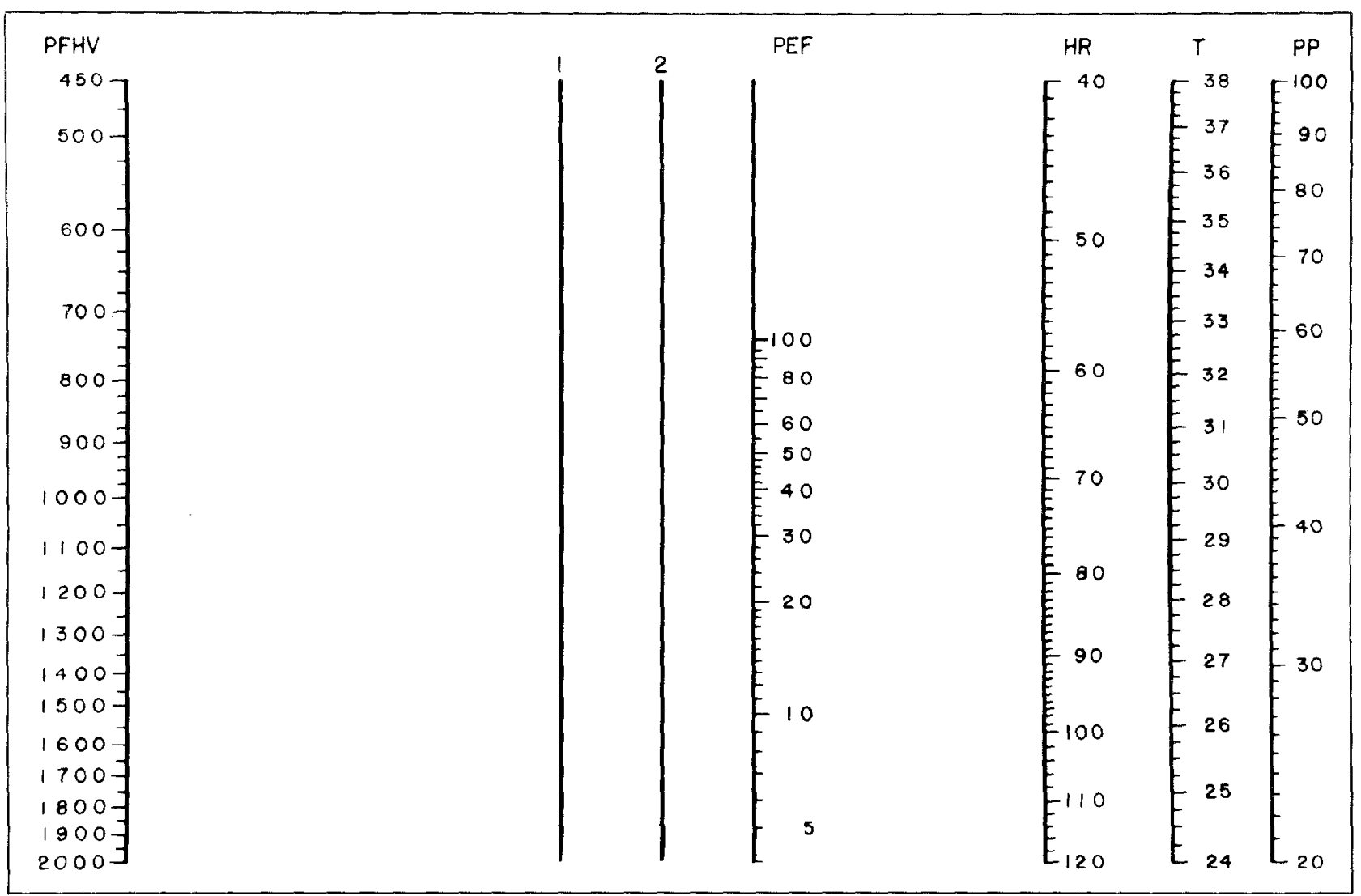

Figure 3. Nomogram for estimation of ejection fraction. The values for plain film heart volume (PFHV and heart rate (HR) are aligned with a straight edge. Pivoting at the intersection with line 1, the straight edge is aligned with the value for the thoracic width (T). Pivoting at the intersection with line 2 , the straight edge is aligned with the value for pulse pressure (PP). The intersection with the PEF scale reveals the value for predicted ejection fraction.

substrata of the exploratory data set is shown in Table I. Predicted and actual ejection fraction was best correlated in patients with no coronary disease $(r=0.84)$. In patients with low-grade, one-, two-, or three-vessel disease, predicted and actual ejection fraction correlated with coefficients between 0.58 and 0.68 . The strength of the correlation appeared unrelated to maximal exercise level. Mild mitral regurgitation did not appear to adversely affect the correlation $(r=0.92)$, but poorer correlation was suggested in the three patients with moderate mitral regurgitation $(r=0.30)$.

Independent Validation. The formula empirically derived in the exploratory data set was subsequently applied to the 41-patient verification data set for independent validation. A plot of predicted and actual ejection fraction is shown in Figure 2. The correlation was found to be higher than in the exploratory data set $(r=0.78)$. Increasing variability with increasing ejection fraction was again noted.

Test Parameters. Test parameters calculated for ejection fractions below 40 are shown in Table IV. The sensitivity was 87 percent, and specificity was 83 per- cent. Predictive values of positive and negative results for 10,50 , and 90 percent pretest probabilities of an ejection fraction below $\mathbf{4 0}$ are given as are the likelihood ratios of positive or negative results.

Nomogram. A nomogram for the graphic calculation of predicted ejection fraction is shown in Figure 3. Scales appear for each independent variable (plain film heart volume, heart rate, pulse pressure, and thoracic width) and the dependent variable (predicted ejection fraction), along with two pivot lines ( 1 and 2). The values for plain film heart volume and heart rate are aligned with a straight edge. Pivoting at the intersection with line 1 , the straight edge is aligned with the value for thoracic width. Pivoting at the intersection with line 2, the straight edge is aligned with the value for pulse pressure. The intersection with the predicted ejection fraction scale reveals the value for predicted ejection fraction.

\section{COMMENTS}

The left ventricular ejection fraction has been established as a valuable prognostic indicator in every major form of cardiac disease including coronary artery disease, valvu- 
lar heart disease, and cardiomyopathies [1-8]. However, determination of the ejection fraction is generally technology-intensive and associated with significant inconvenience, invasiveness, or expense. The pathophysiology that accompanies depression of the ejection fraction would suggest that ventricular function could be assessed with some degree of accuracy using information obtained in routine patient evaluation. Thus, a means of estimating the ejection fraction from simple clinical information would be of both conceptual and practical interest.

Several studies have examined the relationship between the ejection fraction and heart size on routine chest radiography. Plain film heart volume is an established radiographic indicator of heart size [13]. Glover et al [23] showed that while plain film heart volume correlated with left ventricular end-diastolic volume $(r=0.57)$, it correlated best with the sum of left ventricular end-diastolic volume, left ventricular mass, and left atrial volume $(r=$ 0.78 ) in 254 patients with left ventricular pressure overload, volume overload, or both. Chikos et al [24] found similar results in 59 patients with aortic valve disease, although in the 18 normal patients they studied, plain film heart volume was poorly correlated with left ventricular end-diastolic volume or left ventricular end-diastolic volume plus mass, Hammermeister et al [9] subsequently showed that the plain film heart volume was a sensitive but nonspecfic indicator of increased left ventricular enddiastolic volume and decreased ejection fraction in a population with predominant valvular disease. That study also examined intra- and inter-observer variability in plain film heart volume determination and demonstrated standard deviations of 5.2 percent and 7.3 percent, respectively [9]. Accuracy of plain film heart volume has additionally been shown to be independent of observer experience [25]. In each of the studies of plain film heart volme, the cardiothoracic ratio was also examined and found to correlate less well with the variables of interest.

The value of other routine clinical information in the assessment of ventricular function has been examined in groups of patients with acute and chronic coronary artery disease. In many patients following myocardial infarction, a QRS score calculated from routine electrocardiographic results has been shown to correlate with enzymatically or pathologically determined infarct size, exercise capacity, and ejection fraction [10]. The correlation coefficients for ejection fraction and QRS score by this method range from 0.60 to $0.88[10,26]$. However, this method is applicable only in a patient who has had a single myocardial infarction within one year of electrocardiography and who does not have left ventricular hypertrophy, right ventricular hypertrophy, left bundle branch block, right bundle branch block, fasicular block, or intraventricular conduction defect.

Sanford et al [11] examined the utility of data from the history, physical examination, chest radiography, and electrocardiography in the assessment of left ventricular function in 100 patients with acute myocardial infarction and arrived at a six-variable model predicting the ejection fraction with an $R^{2}$ value of 0.42 . The most predictive variables in their study were anterior myocardial infarction, radiographic pulmonary congestion, rales to two thirds of the posterior thorax, previous myocardial infarction, transmural myocardial infarction, and tachycardia. The absence of any heart size assessment from their analysis may, in large part, explain the lower predictive power of their relatively large model. Additionally, use of the QRS score might have improved the contribution of the electrocardiographic results in their population.

Mattleman et al [12] studied 99 patients with coronary artery disease to determine the correlation of routine clinical data with the radionuclide ventriculographic ejection fraction. The four strongest multivariate predictors were radiographic cardiomegaly, $Q$ wave myocardial infarction on electrocardiography, dyspnea, and rales. When combined in a regression model, these predicted the ejection fraction with an $R^{2}$ value of 0.61 ; however, poorer correlation was observed as the ejection fraction decreased. Although clinical and radiographic evidence of pulmonary congestion appeared to contribute significantly to the prediction of the ejection fraction in these studies, we have found-using hemodynamic data from cardiac catheterization rather than radionuclide ventriculography - that left ventricular end-diastolic pressure does not contribute significantly to the multivariate prediction of the ejection fraction when plain film heart volume, heart rate, pulse pressure, and thoracic width are included. The discontinuous and subjective nature of a number of the variables examined in the previous studies may have contributed to the observed variability, although carefully designed clinimetrics and statistical design can enhance the utility of such information [27].

In our study, a general method was sought for predicting left ventricular ejection fraction using simple, quantitative, continuous data from routine patient evaluation. A physiologic model based on fundamental hemodynamic equations was developed a priori and predicted a model with terms multiplied, the nature of possible terms, and the sign of the exponent for each term. These predictions from the general model, in fact, agreed closely with the form of the specific regression formula that optimally predicted the ejection fraction. Logarithmic transformation allowed evaluation of the nonlinear model using standard techniques of multiple linear regression. Comparison with the simpler, but nonphysiologic model using untransformed varlables demonstrated that the theoretically more appropriate physiologically based model provided the more satisfactory mathematical model in practice.

The relationship between the predicted and actual ejection fraction as shown in Figures 1 and 2 demonstrates increasing variability with increasing ejection frac- 
tion. This pattern is, in fact, similar to the pattern of variability for ejection fraction determined by ventriculography. Wackers et al [19] have demonstrated substantially greater variability in the ejection fraction in patients with normal ejection fraction values compared with those with depressed ejection fraction values determined by radionuclide ventriculography. This observation was confirmed by Kaul et al [20] in a larger study and is also seen in the data of Folland et al [21] and Marshall et al [22]. Variability introduced by measurement error or by variation in heart size between systole and diastole would increase with the ejection fraction. The absolute error due to cardiac cycle phase is, however, likely to be small [28].

The study population consisted of stable patients able to undergo routine chest radiography and exercise radionuclide ventriculography. Thus, critically ill patients were not represented in the study, and the method developed is of uncertain relevance to such patients. In addition, the assessments were performed at a single time point for each patient. Therefore, the effect of specific medical or surgical intervention on ejection fraction prediction could not be evaluated in this study.

We suspect that the method will underestimate the ejection fraction in patients with marked aortic or mitral regurgitation, as only a portion of the stroke volume is effective forward stroke volume. In such cases, the predicted ejection fraction may, however, correlate most closely with effective forward ejection fraction. The method is also expected to underestimate the ejection fraction in patients with significant right ventricular enlargement or significant pericardial effusion. Each of these groups can generally be identlfied on cllnical grounds, and In each case, the error is in the direction of underestimating the ejection fraction because of the disproportionately increased plain film heart volume.
Although based on a model postulated a priori, this or any empirically derived formula should be examined in an independent group of patients for validation. A verification data set was developed for this purpose and revealed a similar but even stronger overall correlation between the predicted and actual ejection fraction $(r=0.78)$. The nature of the relationship appeared similar to that seen in the exploratory data. When test parameters were determined for ejection fractions below 40 , useful degrees of sensitivity and specificity were observed.

The method described herein uses simple, objective radiographic measurements and vital signs to predict the ejection fraction using a physiologically based model. The calculations required can be quickly performed using a hand calculator or the nomogram presented. Consequently, the method provides a convenient, noninvasive, and inexpensive means of estimating the left ventricular ejection fraction. Although experienced clinicians may be able to judge left ventricular function with similar accuracy, our method provides an objective, quantitative estimate of ejection fraction that is independent of the expertise of the observer. The clinical assessment of left ventricular function can thus be performed and communicated more effectively in the course of patient management or clinical investigation. Application of this simple method may thus assist physicians in optimizing the use of more invasive and expensive studies.

\section{ACKNOWLEDGMENT}

We are grateful to Dr. Anthony Schork, School of Public Health, University of Michigan, for his careful review of the statistical methods and results and to Ms. Mildred Herman for her excellent assistance in the preparation of the manuscript.

\section{REFERENCES}

1. Schulze RA, Strauss HW, Pitt B: Sudden death in the year following myocardial infarction. Relation to ventricular premature contractions in the late hospital phase and left ventricular ejection fraction. Am $J$ Med 1977; 62: 192-198.

2. Feild BJ, Russell RO, Dowling JT, Rackley CE: Regional left ventricular performance in the year following myocardial infarction. Circulation 1972; 46: 679-689.

3. Norris RM, Barnaby PF, Brandt PWT, et al: Prognosis after recovery from first acute myocardial infarction: determinants of reinfarction and sudden death. Am J Cardiol 1984; 53: 408-413.

4. The Multicenter Postinfarction Research Group: Risk stratification and survival after myocardial infarction. $N$ Engl $J$ Med 1983; 309: 331-336

5. Cohn PF, Gorlin R, Cohn LH, Collins JJ: Left ventricular ejection fraction as a prognostic guide in surgical treatment of coronary and valvular heart disease. Am J Cardiol 1974; 34: 136-141.
6. Hammermeister KE, DeRouen TA, Murray JA, Dodge HT: Effect of aortocoronary saphenous vein bypass grafting on death and sudden death. Am J Cardiol 1977; 39: 925-934.

7. Hammermeister KE, Fisher L, Kennedy JW, Samuels S, Dodge HT: Prediction of late survival in patients with mitral valve disease from clinical, hemodynamic, and quantitative angiographic variables. Circulation 1977; 57 : 341-349.

8. Keats $T E$, Enge IP: Cardiac mensuration by the cardiac volume method. Radiology 1965; 85: 850-855.

9. Feild BJ, Banley WA, Russell RO, et al: Left ventricular function and hypertrophy in cardiomyopathy with depressed ejection fraction. Circulation $1973 ; 47$ : 1022-1031.

10. Hammermeister KE, Chikos PM, Fisher L, Dodge HT: Relationship of cardiothoracic ratio and plain film heart volume to late survival. Circulation 1979; 59: 89-95.

11. Palmeri ST, Harrison DG, Cobb FR, et al: A QRS scoring 
system for assessing left ventricular function after myocardial infarction. N Engl J Med 1982; 306: 4-9.

12. Sanford $C F$, Corbett $J$, Nicod $P$, et al: Value of radionuclide ventriculography in the immediate characterization of patients with acute myocardial infarction. Am $J$ Cardiol 1982; 49: 637-644.

13. Mattleman SJ, Hakki A-H, Iskandrian AS, Segal BL, Kane SA: Reliability of bedside evaluation in determining left ventricular function: correlation with left ventricular ejection fraction determined by radionuclide ventriculography. J Am Coll Cardiol 1983; I: 417-420.

14. Haycock GB, Schwartz GJ, Wisotsky DH: Geometrlc method for measuring body surface area: a height-weight formula validated in infants, children, and adults. J Pediatr 1978; 93: 62-66.

15. Fox DJ, Guire KE: Documentation for MIDAS, 3rd ed. Statistical Research Laboratory, the University of Michigan, University Press, September 1976.

16. University of Michigan Computing Center: MTS, the Michigan Terminal System Volume I: the Michigan Terminal System. Ann Arbor: University of Michigan Computing Center, University Press, February 1983.

17. Ingelfinger JA, Mosteller $F$, Thibodeau $L A$, Ware $\mathrm{JH}$ : Biostatistics in clinical medicine. New York: Macmillan, 1983; $1-32$.

18. Mysiak EJ: Alignment charts. In: Giesecke FE, Mitchel A Spencer HC, Hill IL, Loving RO, Dygdon JT, eds. Engineering graphics, 3rd ed. New York: Macmillan, 1981 743-768.

19. Wackers FJ, Berger HJ, Johnstone DE, et al: Multiple gated cardiac blood pool imaging for left ventricular ejection fraction: validation of the technique and assessment of variability. Am J Cardiol 1979; 43: 1159-1166.

20. Kaul S, Boucher CA, Okada RD, et al: Sources of variability in the radionuclide angiographic assessment of ejection fraction: a comparison of first-pass and gated equilibrium techniques. Am J Cardiol 1984; 53: 823-828.

21. Folland ED, Hamilton GW, Larson SM, Kennedy JW, Williams DL, Ritchie JL: The radionuclide ejection fraction: a comparison of three radionuclide techniques with contrast angiography. J Nucl Med 1977; 18: 1159-1166.

22. Marshall RC, Berger HJ, Reduto LA, Gottschalk A, Zaret BL: Variability in sequential measures of left ventricular performance assessed with radionuclide angiocardiography. Am J Cardiol 1978; 41: 531-536.

23. Glover L, Baxley WA, Dodge HT: A quantitative evaluation of heart size measurements from chest roentgenograms. Circulation 1973; 47: 1289-1296.

24. Chikos PM, Figley MM, Fisher L: Correlation between chest film and angiographic assessment of left ventricular size. AJR 1977; 128: 367-373.

25. Chikos PM, Figley MM, Fisher L: Visual assessment of total heart volume and specific chamber size from standard chest radiographs. AJR 1977; 128: 375-380.

26. Young SG, Abouantoun S, Savvides M, Madsen EB, Froelicher $V$ : Limitations of electrocardiographic scoring system for estimation of left ventricular function. J Am Coll Cardiol 1983; 1: 1479-1488.

27. Feinstein AR: An additional basic science for clinical medicine: IV. The development of clinimetrics. Ann Intern Med 1983; 99: 843-848.

28. Gammill SL, Krebs C, Meyers P, Nice CM, Becker HC: Cardiac measurements in systole and diastole. Radiology 1970; 94: 115-119. 